\title{
Role of fermion exchanges in statistical signatures of composite bosons
}

\author{
M. Combescot, ${ }^{1}$ F. Dubin, ${ }^{2}$ and M. A. Dupertuis ${ }^{3}$ \\ ${ }^{1}$ INSP-Institut des NanoSciences de Paris, Université Pierre et Marie Curie-CNRS, 140 rue de Lourmel, 75015 Paris, France \\ ${ }^{2}$ ICFO-Institut de Ciències Fotòniques, Mediterranean Technology Park, E-08860 Castelldefels, Spain \\ ${ }^{3}$ Laboratoire d'Optoélectronique Quantique et Laboratoire de Physique des Nanostructures, Ecole Polytechnique Fédérale \\ de Lausanne (EPFL), Station 3, CH-1015 Lausanne, Switzerland
}

(Received 10 March 2009; published 21 July 2009)

\begin{abstract}
We study statistical signatures of composite bosons made of two fermions by extending number states to these quantum particles. Two-particle correlations as well as the dispersion of the probability distribution are analyzed. We show that the particle composite nature reduces the antibunching effect predicted for elementary bosons. Furthermore, the probability distribution exhibits a dispersion that is greater for composite bosons than for elementary bosons. This dispersion corresponds to the one of sub-Poissonian processes, as for a quantum state but, unlike its elementary boson counterpart, it is not minimum. In general, our work shows that it is necessary to take into account the Pauli exclusion principle, which acts between fermionic components of composite bosons - along the line used here-to possibly extract statistical properties in a precise way.
\end{abstract}

DOI: $10.1103 /$ PhysRevA.80.013612

PACS number(s): 03.75.-b, 71.35.-y

\section{INTRODUCTION}

Quantum particles obey statistical laws that are highlighted by high-order correlations. This has first been shown for light fields by the discovery of photon bunching in twophoton correlations from an incoherent source [1]. Thereafter, second-order correlations have become increasingly important, particularly to distinguish classical from quantum states of light $[2,3]$. Indeed, classical states are characterized by bunched two-photon correlations while quantum states yield antibunching. For elementary bosons, two-particle correlations are directly connected to the variance of the probability distribution, which is usually expressed through the so-called Mandel parameter $Q$ [4]. The latter compares the dispersion of the distribution to the one of Poissonian processes. Hence, quantum states correspond to negative values of $Q$, i.e., they follow sub-Poissonian statistics, while classical states lead to positive values of $Q$ since these can solely follow Poissonian and super-Poissonian statistics.

Recent advances with ultracold atoms have shown that analysis of second-order correlations is also well suited to probe matter states (see [5] and references therein). Most notably, antibunching and bunching effects have been observed while measuring two-particle correlations of degenerate Fermi and Bose gases [6-8]. In quantum-mechanical terms, these effects are due to interfering amplitudes for the two paths that particles can take to reach the detectors. Intimately, bunching and antibunching reflect the statistics obeyed by the quantum particles: Bose-Einstein statistics imposes addition of the amplitudes, i.e., constructive interference, while Fermi-Dirac statistics yields destructive interference. In the latter case, as for elementary bosons, an antibunching effect signals that the matter state has no classical analog. Note that fermion antibunching was also observed for electrons [9-11] and neutrons [12].

One encounters a more complex situation while studying composite bosons made of two fermions. In an ensemble of such quantum particles, the Pauli exclusion principle induces fermion exchanges between composite bosons, which there- fore do not exactly follow Bose-Einstein statistics. Semiconductor excitons constitute a good example of such composite bosons. These are made of electron-hole pairs and fermion exchanges between the electrons, and holes of these excitons are highlighted by optical nonlinearities [13]. Interacting Fermi gases, made of, e.g., ${ }^{6} \mathrm{Li}$ and ${ }^{40} \mathrm{~K}$, constitute other systems where composite bosons can be studied. In ultracold samples [14], these offer an interesting route toward precise investigation of the Pauli exclusion principle. Indeed, exchanges between fermionic components can be varied, for instance when imbalanced spin populations interact [15].

Recently, a significant breakthrough was made in the theoretical description of composite bosons made of two fermions. A formalism, free from any mapping to an ideal boson subspace, has been constructed, and a visualization of the physical processes taking place between composite bosons (cobosons) has been proposed through the so-called "Shiva" diagrams (see [16] for a general review). This coboson many-body theory relies on two sets of $2 \times 2$ scatterings: the "interaction scatterings" and the "Pauli scatterings." The first ones correspond to interactions between the fermionic components of two cobosons in the absence of fermion exchanges while Pauli scatterings correspond to fermion exchanges between two cobosons, without any fermion interaction.

In this work, we use this many-body approach to study the influence of fermion exchanges in statistical signatures of composite bosons. To highlight corresponding effects, we extend number states to composite bosons by considering a many-body state constructed from $N$ identical coboson creation operators. Hence, we calculate the number of coincidences in two-particle correlations, $g_{N}^{(2)}$, as well as the variance of the field that is evaluated through the Mandel parameter $Q_{N}$. From the corresponding exact expressions we then extract leading terms in density, in the large $N$ limit, in order to quantitatively probe the influence of particle compositeness on these quantum order parameters.

For number states made of elementary bosons, $\left|\bar{\psi}_{N}\right\rangle=\bar{B}_{0}^{\dagger N}|v\rangle$, where $\bar{B}_{0}^{\dagger}$ is the field-particle creation operator 
and $|v\rangle$ is the vacuum state, it is known that $\bar{g}_{N}^{(2)}$ and $\bar{Q}_{N}$ read as $(1-1 / N)$ and $(-1)$, respectively: number states exhibit an antibunched second-order correlation function and yield the greatest negative value for the Mandel parameter. This implies that measurements of such states are made with the greatest sensitivity, i.e., that noise is reduced at the smallest level. For composite bosons in a many-body state $\left|\psi_{N}\right\rangle=B_{0}^{\dagger N}|v\rangle$, we find that fermion exchanges modify the number of coincidences that reads, in the large $N$ but small density limit, $g_{N}^{(2)} \simeq 1+[-1+O(\eta)] / N$. The dimensionless parameter $\eta=N\left(a_{B} / L\right)^{D}$ is associated to the composite boson density, $a_{B}$ being the coboson extension, $L$ the sample size, and $D$ the space dimension. Here, $O(\eta)$ is a positive correction whose dominant term increases linearly with $\eta$. Consequently, two-particle correlations of composite bosons present a reduced antibunching effect when compared to elementary bosons. Furthermore, the amplitude of the $Q$ parameter for the state $\left|\psi_{N}\right\rangle$ reads as $Q_{N} \simeq-1+O\left(\eta^{2}\right)$ in the large $N$ but small $\eta$ limit. Hence, the dispersion of the probability distribution is increased by fermion exchanges. In general, our analysis shows that it is necessary to take into account the Pauli exclusion principle between fermionic components of composite bosons in order to possibly extract statistical properties of these quantum particles in a precise way.

\section{PHYSICAL UNDERSTANDING}

Statistical properties of quantum fields are often studied through the second-order correlation function. The latter evaluates fluctuations in the number of field particles for a given many-body state. Precisely, it measures the normalized probability to detect a particle conditioned upon detection of a previous particle. The normalized number of two-particle coincidences in a state 0 is defined as

$$
g_{N}^{(2)}=\frac{\left\langle B_{0}^{\dagger 2} B_{0}^{2}\right\rangle_{N}}{\left\langle B_{0}^{\dagger} B_{0}\right\rangle_{N}^{2}},
$$

where $\langle A\rangle_{N}=\left\langle\psi_{N}|A| \psi_{N}\right\rangle /\left\langle\psi_{N} \mid \psi_{N}\right\rangle$ is the mean value of the operator $A$ in state $\left|\psi_{N}\right\rangle$, the field-particle creation operator being $B_{0}^{\dagger}$.

For elementary bosons, comparison between the normalized number of coincidences and one allows us to deduce whether fluctuations of the number of particles follow classical or quantum statistics. Another way to study the variance of the number of particles and to characterize a quantum field is via the so-called Mandel parameter, which precisely reads

$$
Q_{N}=\frac{\left\langle\hat{n}^{2}\right\rangle_{N}-\langle\hat{n}\rangle_{N}^{2}}{\langle\hat{n}\rangle_{N}}-1,
$$

where $\hat{n}=B_{0}^{\dagger} B_{0}$ is the number operator for the state 0 .

The parameter $Q_{N}$ compares the fluctuations of the field number operator to that of a Poissonian source [4]. A negative $Q_{N}$ signals that the field statistics is sub-Poissonian and hence corresponds to the one of a quantum state. Most striking examples are obtained for the number states $\left|\bar{\psi}_{N}\right\rangle$ $=\bar{B}_{0}^{\dagger N}|v\rangle$ of particles with bosonic statistics, i.e., $\left[\bar{B}_{m}, \bar{B}_{i}^{\dagger}\right]$ $=\delta_{m i}$. For these, we find $\bar{g}_{N}^{(2)}=(1-1 / N)$ and $\bar{Q}_{N}=-1$ for any particle 0 and any particle density. Note that $(-1)$ is the greatest possible negative value allowed for the Mandel parameter since the ratio in Eq. (2) is always positive: its denominator is the norm of $B_{0}\left|\psi_{N}\right\rangle$ while its numerator is the norm of $P_{\perp} \hat{n}\left|\psi_{N}\right\rangle$, where $P_{\perp}=1-\left|\psi_{N}\right\rangle\left\langle\psi_{N}\right| /\left\langle\psi_{N} \mid \psi_{N}\right\rangle$ is the projector over the subspace perpendicular to $\left|\psi_{N}\right\rangle$, with this operator being such that $P_{\perp}=P_{\perp}^{2}$.

To demonstrate that fermion exchanges noticeably affect statistical signatures of composite bosons, we extend boson number states to cobosons, and evaluate $g_{N}^{(2)}$ and $Q_{N}$ in the state $\left|\psi_{N}\right\rangle=B_{0}^{\dagger N}|v\rangle$. To understand in a simple way of how fermionic components modify these two quantities, we start with $N=2$ since most of the physical effects induced by the Pauli exclusion principle already appear in the extreme dilute limit with just two composite particles.

\section{A. Two composite bosons}

Since $B_{0}^{2}\left|\psi_{2}\right\rangle$ is a zero-pair state, $\left\langle\psi_{2}\left|B_{0}^{\dagger 2} B_{0}^{2}\right| \psi_{2}\right\rangle$ is equal to $\left\langle\psi_{2}\left|B_{0}^{\dagger 2}\right| v\right\rangle\left\langle v\left|B_{0}^{2}\right| \psi_{2}\right\rangle$. As $\left\langle v\left|B_{0}^{2}\right| \psi_{2}\right\rangle$ is nothing but $\left\langle\psi_{2} \mid \psi_{2}\right\rangle$, the normalized two-particle coincidences reduce to

$$
g_{2}^{(2)}=\frac{\left\langle\psi_{2} \mid \psi_{2}\right\rangle^{3}}{\left\langle\psi_{2}|\hat{n}| \psi_{2}\right\rangle^{2}} .
$$

To better see the differences induced by the particle composite nature, let us briefly reconsider elementary bosons, i.e., bosons such that $\left[\bar{B}_{m}, \bar{B}_{i}^{\dagger}\right]=\delta_{m i}$. We then have $\bar{B}_{0}\left|\bar{\psi}_{2}\right\rangle$ $=2\left|\bar{\psi}_{1}\right\rangle$, so that $\bar{B}_{0}^{2}\left|\bar{\psi}_{2}\right\rangle=2|v\rangle$ and $\hat{n}\left|\bar{\psi}_{2}\right\rangle=2\left|\bar{\psi}_{2}\right\rangle$. Consequently $\left\langle\bar{\psi}_{2} \mid \bar{\psi}_{2}\right\rangle=2$ while $\left\langle\bar{\psi}_{2}|\hat{n}| \bar{\psi}_{2}\right\rangle=4$ and $\left\langle\bar{\psi}_{2}\left|\hat{n}^{2}\right| \bar{\psi}_{2}\right\rangle=8$. This leads to the expected results, namely, $\bar{Q}_{2}=-1$ and $\bar{g}_{2}^{(2)}=1 / 2$, which is nothing but $(1-1 / N)$ taken for $N=2$.

We now turn to composite bosons. These have creation operators that do not exactly follow bosonic commutation rules [16] but instead

$$
\left[B_{m}, B_{i}^{\dagger}\right]=\delta_{m i}-D_{m i},
$$

where the so-called "deviation-from-boson" operator, $D_{m i}$, is such that $D_{m i}|v\rangle=0$ while

$$
\left[D_{m i}, B_{j}^{\dagger}\right]=\sum_{n}\left[\lambda\left(\begin{array}{ll}
n & i \\
m & j
\end{array}\right)+i \leftrightarrow j\right] B_{n}^{\dagger} .
$$

The parameters $\lambda\left(\begin{array}{cc}n & i \\ m & j\end{array}\right)$ are the composite bosons "Pauli scatterings"; they describe fermion exchanges between $(i, j)$ in the absence of fermion interaction [see Fig. 1(a)].

The previous commutators give $B_{0}\left|\psi_{2}\right\rangle=2\left|\psi_{1}\right\rangle-2 L_{2}^{\dagger}|v\rangle$ and $B_{0} L_{2}^{\dagger}\left|\psi_{1}\right\rangle=\lambda_{2}\left|\psi_{1}\right\rangle+\left(L_{2}^{\dagger}-2 L_{3}^{\dagger}\right)|v\rangle$, where we have set $\lambda_{2}$ $=\lambda\left(\begin{array}{ll}0 & 0 \\ 0 & 0\end{array}\right)$ while

$$
\begin{gathered}
L_{2}^{\dagger}=\frac{1}{2}\left[D_{00}, B_{0}^{\dagger}\right]=\sum_{n} \lambda\left(\begin{array}{ll}
n & 0 \\
0 & 0
\end{array}\right) B_{n}^{\dagger}, \\
L_{3}^{\dagger}=\frac{1}{2}\left[D_{00}, L_{2}^{\dagger}\right]=\sum_{n, p} \lambda\left(\begin{array}{ll}
p & 0 \\
0 & n
\end{array}\right) \lambda\left(\begin{array}{ll}
n & 0 \\
0 & 0
\end{array}\right) B_{p}^{\dagger} .
\end{gathered}
$$

This allows one to find $B_{0}^{2}\left|\psi_{2}\right\rangle=2\left(1-\lambda_{2}\right)|v\rangle$, which gives $\left\langle\psi_{2} \mid \psi_{2}\right\rangle=2\left(1-\lambda_{2}\right)$. This also leads to $\hat{n}\left|\psi_{2}\right\rangle=2\left|\psi_{2}\right\rangle-2 L_{2}^{\dagger}\left|\psi_{1}\right\rangle$. 


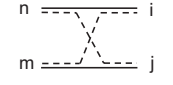

(a)
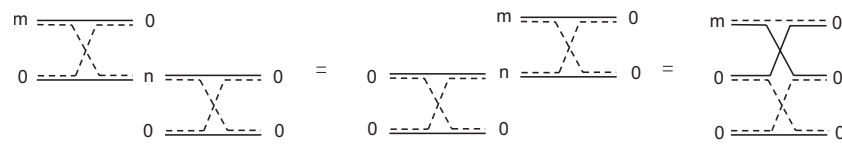

(b)

FIG. 1. Shiva diagrams representing fermion exchanges between composite bosons. Fermionic components are represented by solid and dashed lines. (a) Fermion exchange between two "in" cobosons in states $(i, j)$ leading to the formation of two "out" cobosons in states $(n, m)$. This diagram represents the Pauli scattering $\lambda\left(\begin{array}{ll}n & i \\ m & j\end{array}\right)$. (b) Fermion exchange between three "in" cobosons 0 yielding three "out" cobosons $(m, 0,0)$, as appearing in Eqs. (7) and (A8). For $m=0$, this exchange corresponds to the three-body scattering $\lambda_{3}=\Sigma_{n} \lambda\left(\begin{array}{ll}0 & 0 \\ 0 & n\end{array}\right) \lambda\left(\begin{array}{cc}n & 0 \\ 0 & 0\end{array}\right)$ of Eq. (8).

In order to calculate $\left\langle\psi_{2}|\hat{n}| \psi_{2}\right\rangle$ in an easy way, it is convenient to first note that $\left\langle\psi_{2}\left|L_{2}^{\dagger}\right| \psi_{1}\right\rangle=\left\langle\psi_{2}\left|B_{0}^{\dagger} L_{2}^{\dagger}\right| v\right\rangle$. From $\left\langle\psi_{2}\right| B_{0}^{\dagger}$ as given above, we then find that $\left\langle\psi_{2}\left|L_{2}^{\dagger}\right| \psi_{1}\right\rangle=2\left(\lambda_{2}-\lambda_{3}\right)$, where $\lambda_{3}=\Sigma_{n} \lambda\left(\begin{array}{ll}0 & 0 \\ 0 & n\end{array}\right) \lambda\left(\begin{array}{ll}n & 0 \\ 0 & 0\end{array}\right)$ corresponds to the Shiva diagram for fermion exchanges between three composite bosons 0 , shown in Fig. 1(b). All this leads to

$$
\left\langle\psi_{2}|\hat{n}| \psi_{2}\right\rangle=4\left(1-2 \lambda_{2}+\lambda_{3}\right)
$$

The most convenient way to obtain $\left\langle\psi_{2}\left|\hat{n}^{2}\right| \psi_{2}\right\rangle$ is to first calculate $B_{0} \hat{n}\left|\psi_{2}\right\rangle$ knowing $B_{0} L_{2}^{\dagger}\left|\psi_{1}\right\rangle$ given above. By noting that $\left\langle\psi_{2}\left|L_{3}^{\dagger}\right| \psi_{1}\right\rangle=2\left(\lambda_{3}-\lambda_{4}\right)$, where $\lambda_{4}$ corresponds to the Shiva diagram for fermion exchanges between four composite bosons 0 , we end with

$$
\left\langle\psi_{2}\left|\hat{n}^{2}\right| \psi_{2}\right\rangle=4\left(2-6 \lambda_{2}+\lambda_{2}^{2}+5 \lambda_{3}-2 \lambda_{4}\right) .
$$

Before going further, we can check that the above results for cobosons reduce to the ones for elementary bosons when all fermion exchanges, i.e., all $\lambda_{n}$ 's, are dropped.

For cobosons 0 with center-of-mass momentum $\mathbf{Q}_{0}$ and relative motion index $\nu_{0}$, it is possible to show [16] that $\lambda_{n}$ $=\Sigma_{\mathbf{k}}\left|\left\langle\mathbf{k} \mid \nu_{0}\right\rangle\right|^{2 n}$. This makes $\lambda_{n}$ a positive constant of the order of $\left(a_{B} / L\right)^{(n-1) D}$, where $a_{B}$ is the spatial extension of the relative motion state $\left|\nu_{0}\right\rangle$ and $D$ is the space dimension. For $3 \mathrm{D}$ excitons or hydrogen atoms, for which $\left|\left\langle\mathbf{k} \mid \nu_{0}\right\rangle\right|^{2}$ $=64 \pi\left(a_{B} / L\right)^{3} /\left(1+k^{2} a_{B}^{2}\right)^{2}$, one can show [16] that

$$
\lambda_{n}=16 \frac{(8 n-5) ! !}{(8 n-2) ! !}\left(\frac{64 \pi a_{B}^{3}}{L^{3}}\right)^{n-1} .
$$

This leads to $\lambda_{2}=(33 \pi / 2)\left(a_{B} / L\right)^{3}$ and $\lambda_{3}$ $=\left(4199 \pi^{2} / 8\right)\left(a_{B} / L\right)^{6}$, which makes $\lambda_{3} \ll \lambda_{2}$ and $\left(\lambda_{3}-\lambda_{2}^{2}\right)$, as appearing below in the Mandel parameter, a positive constant equal to $\left(2021 \pi^{2} / 8\right)\left(a_{B} / L\right)^{6}$.

By collecting all the above results, we end with a normalized number for two-particle coincidences of composite bosons, which reads as

$$
g_{2}^{(2)}=\frac{1}{2} \frac{\left(1-\lambda_{2}\right)^{3}}{\left(1-2 \lambda_{2}+\lambda_{3}\right)^{2}} \simeq \frac{1}{2}\left(1+\lambda_{2}\right),
$$

at leading order in $\left(a_{B} / L\right)^{D}$ while the Mandel parameter is given by

$$
Q_{2} \approx-1+\left(\lambda_{3}-\lambda_{2}^{2}\right) \text {. }
$$

This shows that, for $N=2$, the number of two-particle coincidences is slightly higher for composite bosons than for elementary ones. We also find that the Mandel parameter is slightly increased. As seen from Eqs. (11) and (12), the corresponding variations are solely controlled by the Pauli exclusion principle, through the exchange scatterings $\lambda_{n}$ between $n$ cobosons 0 . Unlike elementary bosons for which the second-order correlation function and the Mandel parameter depend on $N$ only, their counterparts for composite bosons also depend on the state 0 at hand, through its spatial extension $a_{B}$.

\section{B. Guess for $N$ composite bosons}

For $N$ elementary bosons, number states are such that $\hat{n}\left|\bar{\psi}_{N}\right\rangle=N\left|\bar{\psi}_{N}\right\rangle$ such that $\bar{g}_{N}^{(2)}=1-1 / N$ and $\bar{Q}_{N}=-1$ for all $N$, whatever the state 0 is.

For composite bosons, a "rule of the thumb" intuitively gives the leading term in the small density limit, through the replacement of $\left(a_{B} / L\right)^{D}$ by $(N-1)\left(a_{B} / L\right)^{D}$ in the result for $N=2$. Since $\lambda_{3}$ and $\lambda_{2}^{2}$ are both of the order of $\left(a_{B} / L\right)^{D}$, this leads us to guess that the Mandel parameter dominant term in density reads as

$$
Q_{N} \simeq-1+(N-1)^{2}\left(\lambda_{3}-\lambda_{2}^{2}\right) \simeq-1+O\left(\eta^{2}\right),
$$

where $\eta$ is the previously defined dimensionless parameter associated to density.

The situation for $g_{N}^{(2)}$ is more ambiguous. From Eq. (11), we might guess that

$$
g_{N}^{(2)} \approx\left(1-\frac{1}{N}\right)\left[1+(N-1) \lambda_{2}\right] \approx\left(1-\frac{1}{N}\right)[1+O(\eta)] .
$$

This would lead to a crossover from antibunching to bunching when $1 / N$ crosses $\eta$, i.e., when the system size increases - which is very unlikely, physically. However, we might as well guess

$$
g_{N}^{(2)} \simeq 1+\frac{-1+(N-1) \lambda_{2}}{N} \simeq 1+\frac{-1+O(\eta)}{N},
$$

which maintains the antibunching effect for all sample sizes since the $\lambda_{n}$ expansions performed in these calculations only hold for $\eta \ll 1$. The purpose of the next section is to demonstrate that Eqs. (13) and (15) are indeed the correct expressions of $Q_{N}$ and $g_{N}^{(2)}$. To do it, we must go deeper into the composite boson many-body theory. 


\section{SECOND-ORDER CORRELATIONS FOR $\boldsymbol{N}$ COMPOSITE BOSONS}

\section{A. Key results from the coboson many-body theory}

Many-body effects between $N$ composite bosons linked to fermion exchanges are obtained through the two following commutators [16]

$$
\begin{gathered}
{\left[B_{m}, B_{i}^{\dagger N}\right]=} \\
-N B_{i}^{\dagger N-1}\left(\delta_{m i}-D_{m i}\right) \\
-N-1) B_{i}^{\dagger N-2} \sum_{n} \lambda\left(\begin{array}{cc}
n & i \\
m & i
\end{array}\right) B_{n}^{\dagger}, \\
{\left[D_{m i}, B_{j}^{\dagger N}\right]=N B_{j}^{\dagger N-1} \sum_{n}\left[\lambda\left(\begin{array}{ll}
n & j \\
m & i
\end{array}\right)+\lambda\left(\begin{array}{ll}
n & i \\
m & j
\end{array}\right)\right] B_{n}^{\dagger} .}
\end{gathered}
$$

These two commutators reduce to Eqs. (4) and (5) when $N=1$. Using them, it is possible to show that the normalization factor for $N$ cobosons in the same 0 state differs from its elementary bosons value $(N$ !) due to fermion exchanges taking place between them. This led us to write

$$
\left\langle v\left|B_{0}^{N} B_{0}^{\dagger N}\right| v\right\rangle=N ! F_{N},
$$

where the $F_{N}$ 's, which enter all calculations involving a large number of identical cobosons, follow the recursion relation

$$
\begin{aligned}
F_{N} & =F_{N-1}-(N-1) \lambda_{2} F_{N-2}+(N-1)(N-2) \lambda_{3} F_{N-3}-\cdots \\
& =\sum_{n}(-1)^{n-1} \frac{(N-1) !}{(N-n) !} \lambda_{n} F_{N-n} .
\end{aligned}
$$

The $\lambda_{n}$ 's, shown in Fig. 1 for $n=2$ or 3 , are the previously defined scatterings for fermion exchanges between $n$ cobosons 0 .

Equation (16) readily shows that

$$
B_{0}\left|\psi_{N}\right\rangle=N\left|\psi_{N-1}\right\rangle-N(N-1) L_{2}^{\dagger}\left|\psi_{N-2}\right\rangle,
$$

with $L_{2}^{\dagger}$ given in Eq. (6). The above equation used for $B_{0}\left|\psi_{N}\right\rangle$ and then for $L_{2}^{\dagger}\left|\psi_{N-1}\right\rangle$ allows us to show that

$$
B_{0}^{\dagger} B_{0}\left|\psi_{N}\right\rangle=\left|\psi_{N}\right\rangle+\frac{N-1}{N+1} B_{0}\left|\psi_{N+1}\right\rangle
$$

This last equation will turn very useful in calculating matrix elements.

\section{B. Calculation of $Q_{N}$}

Since $\left\langle\psi_{N}\right| B_{0}=\left\langle\psi_{N+1}\right|$, Eqs. (18) and (21) give the mean value of the particle 0 number operator as

$$
\langle\hat{n}\rangle_{N}=1+(N-1) \frac{F_{N+1}}{F_{N}}=N+(N-1) \Delta_{N}^{(1)},
$$

where we have set $\Delta_{N}^{(n)}=\left(F_{N+n}-F_{N+n-1}\right) / F_{N}$.

For $N=2$ cobosons, Eq. (19) gives $F_{2}=1-\lambda_{2}$ and $F_{3}=1$ $-3 \lambda_{2}+2 \lambda_{3}$, so that we recover Eq. (8). For $N$ elementary bosons, $F_{N}$ reduces to one so that all the $\Delta_{N}^{(n)}$ differences reduce to zero. Hence, the number-operator mean value is equal to $N$, as expected. By contrast, $\Delta_{N}^{(n)}$ for composite bosons are negative scalars since $F_{N}$ is a decreasing function of $N$, as seen from Eq. (19). This makes the number-operator mean value for cobosons smaller than its elementary boson value $N$ due to a "moth-eaten" effect similar to the one we have already found in other problems dealing with composite bosons: when a coboson 0 is added to $N$ other cobosons 0 , the additional coboson feels the other $N$ 's through the Pauli exclusion principle. Therefore $N$ elementary fermion pair states are blocked and thus missing in its linear combination, as if these were "eaten" by $N$ "moths." This informal picture allows us to physically understand all decreases found for cobosons, when compared to their counterpart elementary boson values.

By repeatedly using Eq. (21) for $\left\langle\psi_{N}\left|\hat{n}^{2}\right| \psi_{N}\right\rangle$ split as $\left(\left\langle\psi_{N}\right| \hat{n}\right)\left(\hat{n}\left|\psi_{N}\right\rangle\right)$, we can show that

$$
\left\langle\hat{n}^{2}\right\rangle_{N}=N^{2}+\left(N^{2}-1\right) \Delta_{N}^{(1)}+\frac{N(N-1)^{2}}{N+1} \Delta_{N}^{(2)} .
$$

Again, $\left\langle\hat{n}^{2}\right\rangle_{N}$ for cobosons is smaller than its $N^{2}$ value for elementary bosons due to the same moth-eaten effect since all the $\Delta_{N}^{(n)}$ are negative. Using $F_{4}=1-6 \lambda_{2}+8 \lambda_{3}+3 \lambda_{2}^{2}-6 \lambda_{4}$, as deduced from Eq. (19), it is possible to check that the above equation does agree with Eq. (9) when $N=2$.

The two above equations allow us to write the mean quadratic deviation in a compact form in terms of two $F_{N}$ differences only, namely,

$$
\left\langle\hat{n}^{2}\right\rangle_{N}-\langle\hat{n}\rangle_{N}^{2}=(N-1)^{2}\left[-\Delta_{N}^{(1)}\left(1+\Delta_{N}^{(1)}\right)+\frac{N}{N+1} \Delta_{N}^{(2)}\right] .
$$

Since, in the small density limit, $\Delta_{N}^{(1)} \approx-N \lambda_{2}+N(N-1)\left(\lambda_{3}\right.$ $\left.-\lambda_{2}^{2}\right)$ and $\Delta_{N}^{(2)} \approx-(N+1) \lambda_{2}+(N+1) N \lambda_{3}$, as deduced from Eq. (19), we find that the mean quadratic deviation has no term in $\left(a_{B} / L\right)^{D}$, its small density dominant term being $N(N$ $-1)^{2}\left(\lambda_{3}-\lambda_{2}^{2}\right)$. Using Eq. (22), it is then easy to show that the leading correction of $Q_{N}$ given in Eq. (13), as guessed from the rule of the thumb, is fully correct: the $\eta$ term cancels, making $Q_{N}$ increase quadratically with $\eta$.

\section{Calculation of $g_{N}^{(2)}$}

The calculation of the normalized number of coincidences is a little more demanding. To evaluate the numerator of $g_{N}^{(2)}$, we first rewrite $B_{0}^{\dagger} B_{0}$ using commutator (4). This yields

$$
\left\langle B_{0}^{\dagger}\left(B_{0}^{\dagger} B_{0}\right) B_{0}^{\dagger}\right\rangle_{N}=\left\langle\hat{n}^{2}-\hat{n}\right\rangle_{N}+\left\langle B_{0}^{\dagger} D_{00} B_{0}\right\rangle_{N}
$$

The first term readily follows from Eqs. (22) and (23). However, nontrivial manipulations are necessary to get the second term in a compact form. Otherwise, we end with $F_{N}$ expansions that are far from obvious to sum up in terms of three $F_{N}$ only, namely, $F_{N}, F_{N+1}$, and $F_{N+2}$, as it ends by reading. These manipulations are reported in the Appendix. They lead to 


$$
\begin{aligned}
\left\langle B_{0}^{\dagger} D_{00} B_{0}\right\rangle_{N} & =-2\left[\lambda_{2}+\Delta_{N}^{(1)}+\frac{(N-1)^{2}}{N+1} \Delta_{N}^{(2)}\right] \\
& =-2 N(N-1)\left(\frac{\Delta_{N}^{(2)}}{N+1}-R\right),
\end{aligned}
$$

where $R$, defined as

$$
R=\frac{1}{N} \frac{\Delta_{N}^{(2)}}{N+1}-\frac{1}{N(N-1)}\left(\lambda_{2}+\Delta_{N}^{(1)}\right),
$$

tends to $\lambda_{2}^{2}$ in the large sample limit (see Appendix).

Using Eqs. (22), (23), (25), and (26), it is then possible to show that

$$
\left\langle B_{0}^{\dagger 2} B_{0}^{2}\right\rangle_{N}=N(N-1)\left[1+\Delta_{N}^{(1)}+\frac{N-3}{N+1} \Delta_{N}^{(2)}+2 R\right] .
$$

The above equation shows that $g_{N}^{(2)}$ reads in a compact form in terms of $F_{N}, F_{N+1}$, and $F_{N+2}$ only. To determine the dominant contribution in the large sample limit, one can remember that $\Delta_{N}^{(1)}$ tends to $-N \lambda_{2}$ and $\Delta_{N}^{(2)}$ to $-(N+1) \lambda_{2}$ while $R$ tends to $\lambda_{2}^{2}$ in this limit. We then find that the ratio in the above equation tends to $\left[1-(2 N-3) \lambda_{2}\right] /\left[1-(N-1) \lambda_{2}\right]$ so that we end with

$$
g_{N}^{(2)}=\left(1-\frac{1}{N}\right) \frac{1+\Delta_{N}^{(1)}+\frac{N-3}{N+1} \Delta_{N}^{(2)}+2 R}{\left(1+\frac{N-1}{N} \Delta_{N}^{(1)}\right)^{2}},
$$

which, in the small density but large $N$ limit, reads as

$$
g_{N}^{(2)} \approx\left(1-\frac{1}{N}\right)\left(1+\lambda_{2}\right) \approx 1+\frac{-1+(N-1) \lambda_{2}}{N},
$$

in agreement with the result guessed in Eq. (15). Since $\lambda_{2}$ is in $\left(a_{B} / L\right)^{D}$, this shows that the correction induced by bosons composite nature is in $\eta$ for $g_{N}^{(2)}$ but in $\eta^{2}$ for the Mandel parameter $Q_{N}$.

\section{CONCLUSIONS}

By extending number states to composite bosons, we have shown that the Pauli exclusion principle modifies statistical signatures of composite boson many-body quantum states. This is underlined through the particular evaluation of the number of coincidences in two-particle correlations and the dispersion of the corresponding probability distribution. These explicitly reflect fermion exchanges between components of composite bosons. We find that the number of coincidences in second-order correlations is enhanced compared to elementary bosons and so is the variance of the field. However, main statistical signatures are found to be preserved. Indeed, the extension of number states to composite bosons still presents a strong quantum character, with a Mandel $Q$ parameter close to $(-1)$.

To highlight these effects in the simplest way, we have first considered $N=2$ composite bosons. From results obtained for the number of coincidences in two-particle correlations as well as the $Q$ parameter, we used a rather intuitive rule of the thumb to determine these for arbitrary $N$. The corresponding expressions, guessed from the $N=2$ results, are thereafter confirmed by $N$-body calculations. These are done following a procedure proposed in Ref. [16] to handle fermion exchanges between composite bosons, exactly.

In general, our analysis confirms that the statistics of bosonic fields is modified by the underlying fermionic components of bosons. Precisely, fermion exchanges correlate composite boson states through Pauli exclusion principle. This, in particular, makes the mean value of the number operator smaller for composite bosons than for elementary ones. Therefore, coherence properties of composite bosons cannot be directly deduced from their elementary boson counterparts. For that purpose, the derivation of operators peculiar to composite bosons, in order to possibly define "number states" and "coherent states," would be highly valuable.

\section{APPENDIX: EVALUATION OF $\left\langle B_{0}^{\dagger} D_{00} B_{0}\right\rangle_{N}$}

This appendix is dedicated to the calculation of $\left\langle B_{0}^{\dagger} D_{00} B_{0}\right\rangle_{N}$ in a compact form. For that, we first use commutator (5). This allows us to write

$$
\left\langle B_{0}^{\dagger} D_{00} B_{0}\right\rangle_{N}=\left\langle D_{00} B_{0}^{\dagger} B_{0}\right\rangle_{N}-2\left\langle L_{2}^{\dagger} B_{0}\right\rangle_{N} .
$$

The part $\left\langle\psi_{N}\right| D_{00}$ in the first term is obtained by mixing Eqs. (17) and (20). This leads to

$$
D_{00}\left|\psi_{N}\right\rangle=2 N L_{2}^{\dagger}\left|\psi_{N-1}\right\rangle=2\left|\psi_{N}\right\rangle-\frac{2}{N+1} B_{0}\left|\psi_{N+1}\right\rangle .
$$

By using Eq. (21), it is then easy to show that the first term of Eq. (A1) is equal to

$$
\left\langle D_{00} B_{0}^{\dagger} B_{0}\right\rangle_{N}=-2 \Delta_{N}^{(1)}-2 \frac{N(N-1)}{N+1} \Delta_{N}^{(2)} .
$$

In the second term of Eq. (A1), we use Eq. (20) for $B_{0}\left|\psi_{N}\right\rangle$ and then the same Eq. (20), but for $L_{2}^{\dagger}\left|\psi_{N-1}\right\rangle$. This leads to

$$
\left\langle L_{2}^{\dagger} B_{0}\right\rangle_{N}=-\Delta_{N}^{(1)}-N(N-1) R,
$$

where $R$ is defined by

$$
R=\frac{\left\langle\psi_{N}\left|L_{2}^{\dagger 2}\right| \psi_{N-2}\right\rangle_{N}}{\left\langle\psi_{N} \mid \psi_{N}\right\rangle_{N}}
$$

All this allows us to rewrite Eq. (A1) as in Eq. (26).

To get $R$ in a compact form is considerably more difficult. Since it contains two Pauli scattering at least, through the two $L_{2}^{\dagger}$ operators, we can already say that its leading term must be in $\left(a_{B} / L\right)^{2 D}$. This is going to give a $\eta^{2}$ contribution to $g_{N}^{(2)}$, negligible compared to the dominant term of $g_{N}^{(2)}$, expected to be in $\eta$. Nevertheless, for completeness let us 
now show how a compact expression of $R$ can be obtained.

The trick is to note that, for $L_{2}^{\dagger}$ given in Eq. (6), we have, using Eq. (16)

$$
\begin{aligned}
L_{2}\left|\psi_{N}\right\rangle= & \sum_{n} \lambda\left(\begin{array}{cc}
0 & n \\
0 & 0
\end{array}\right)\left[N \delta_{n 0}\left|\psi_{N-1}\right\rangle\right. \\
& \left.-N(N-1) \sum_{m} \lambda\left(\begin{array}{cc}
m & 0 \\
n & 0
\end{array}\right) B_{m}^{\dagger}\left|\psi_{N-2}\right\rangle\right] \\
= & N \lambda_{2}\left|\psi_{N-1}\right\rangle-N(N-1) L_{3}^{\dagger}\left|\psi_{N-2}\right\rangle,
\end{aligned}
$$

where $L_{3}^{\dagger}$ is nothing but the operator defined in Eq. (7) since $\Sigma \lambda\left(\begin{array}{ll}0 & n \\ 0 & 0\end{array}\right) \lambda\left(\begin{array}{ll}m & 0 \\ n & 0\end{array}\right)$ is the three-body exchange scattering with three cobosons 0 on the right and two cobosons 0 plus one coboson $m$ on the left, as readily seen from Fig. 1(b). By using the above equation for $\left\langle\psi_{N}\right| L_{2}^{\dagger}$ but Eq. (20) for $L_{2}^{\dagger}\left|\psi_{N}\right\rangle$, it becomes easy to show that

$$
R=\frac{\lambda_{2}}{N-1} \frac{F_{N-1}-F_{N}}{F_{N}}+\frac{\left\langle\psi_{N-1}\left|L_{3}\right| \psi_{N}\right\rangle-N\left\langle\psi_{N-2}\left|L_{3}\right| \psi_{N-1}\right\rangle}{\left\langle\psi_{N} \mid \psi_{N}\right\rangle}
$$

The next step is to calculate $\left\langle\psi_{N-2}\left|L_{3}\right| \psi_{N-1}\right\rangle$. This is done by again using Eq. (A6) for $\left\langle\psi_{N-2}\right| L_{3}$ and then Eq. (20) for $L_{2}^{\dagger}\left|\psi_{N-1}\right\rangle$. This leads to

$$
\begin{aligned}
\left\langle\psi_{N-2}\left|L_{3}\right| \psi_{N-1}\right\rangle= & \frac{\lambda_{2}}{N-1}\left\langle\psi_{N-1} \mid \psi_{N-1}\right\rangle \\
& -\frac{1}{N^{2}(N-1)}\left\langle\psi_{N} \mid \psi_{N}\right\rangle \\
& +\frac{1}{N^{2}\left(N^{2}-1\right)}\left\langle\psi_{N+1} \mid \psi_{N+1}\right\rangle,
\end{aligned}
$$

so that we ultimately find the expression of $R$ given in Eq. (27). By using the values of $\Delta_{N}^{(1)}$ and $\Delta_{N}^{(2)}$ in the large sample limit, given above, one can show that $R \simeq \lambda_{2}^{2}$. The correction to $\left\langle B_{0}^{\dagger} D_{00} B_{0}\right\rangle_{N}$ induced by this $R$ term is thus found to be of the order of $\eta^{2}$, as expected.
[1] R. Hanbury Brown and R. Q. Twiss, Nature (London) 178, 1447 (1956).

[2] L. Mandel and E. Wolf, Optical Coherence and Quantum Optics (Cambridge University Press, New York, 1995).

[3] M. O. Scully and M. S. Zubairy, Quantum Optics (Cambridge University Press, New York, 1997).

[4] L. Mandel, Opt. Lett. 4, 205 (1979).

[5] I. Bloch, J. Dalibard, and W. Zwerger, Rev. Mod. Phys. 80, 885 (2008)

[6] S. Fölling, F. Gerbier, A. Widera, O. Mandel, T. Gericke, and I. Bloch, Nature (London) 434, 481 (2005).

[7] T. Rom, Th. Best, D. van Oosten, U. Schneider, S. Fölling, B. Paredes, and I. Bloch, Nature (London) 444, 733 (2006).

[8] T. Jeltes, J. M. McNamara, W. Hogervorst, W. Vassen, V. Krachmalnicoff, M. Schellekens, A. Perrin, H. Chang, D. Boiron, A. Aspect, and C. I. Westbrook, Nature (London) 445, 402 (2007).

[9] W. D. Oliver, J. Kim, R. C. Liu, and Y. Yamamoto, Science 284, 299 (1999).

[10] M. Henny, S. Oberholzer, C. Strunk, T. Heinzel, K. Ensslin,
M. Holland, and C. Schoenenberger, Science 284, 296 (1999).

[11] H. Kiesel, A. Renz, and F. Hasselbach, Nature (London) 418, 392 (2002).

[12] M. Iannuzzi, A. Orecchini, F. Sacchetti, P. Facchi, and S. Pascazio, Phys. Rev. Lett. 96, 080402 (2006).

[13] M. Combescot, O. Betbeder-Matibet, K. Cho, and H. Ajiki, Europhys. Lett. 72, 618 (2005); M. Combescot and O. Betbeder-Matibet, Phys. Rev. B 74, 125316 (2006).

[14] S. Jochim, M. Bartenstein, A. Altmeyer, G. Hendl, S. Riedl, C. Chin, J. Hecker Denschlag, and R. Grimm, Science 302, 2101 (2003); M. Greiner, C. A. Regal, and D. S. Jin, Nature (London) 426, 537 (2003); M. W. Zwierlein, C. A. Stan, C. H. Schunck, S. M. F. Raupach, S. Gupta, Z. Hadzibabic, and W. Ketterle, Phys. Rev. Lett. 91, 250401 (2003).

[15] M. W. Zwierlein, A. Schirotzek, C. H. Schunck, and W. Ketterle, Science 311, 492 (2006); G. B. Partridge, W. Li, R. I. Kamar, Y. Liao, and R. G. Hulet, ibid. 311, 503 (2006).

[16] M. Combescot, O. Betbeder-Matibet, and F. Dubin, Phys. Rep. 463, 215 (2008). 